\title{
Performance Characterization of the 64-bit x86 Architecture from Compiler Optimizations' Perspective
}

\author{
Jack Liu and Youfeng Wu \\ Intel Corporation, \\ 2200 Mission Blvd, Santa Clara, CA \\ \{jack.liu, youfeng.wu\} aintel.com \\ http: //www. intel.com/
}

\begin{abstract}
Intel Extended Memory 64 Technology (EM64T) and AMD 64-bit architecture (AMD64) are emerging 64-bit x86 architectures that are fully x86 compatible. Compared with the 32-bit x86 architecture, the 64-bit x86 architectures cater some new features to applications. For instance, applications can address 64 bits of virtual memory space, perform operations on 64-bit-wide operands, get access to 16 general-purpose registers (GPRs) and 16 extended multi-media (XMM) registers, and use a register-based argument passing convention. In this paper, we investigate the performance impacts of these new features from compiler optimizations' standpoint. Our research compiler is based on the Intel Fortran/C++ production compiler, and our experiments are conducted on the SPEC2000 benchmark suite. Results show that for 64-bit-wide pointer and long data types, several SPEC2000 C benchmarks are slowed down by more than $20 \%$, which is mainly due to the enlarged memory footprint. To evaluate the performance potential of 64-bit x 86 architectures, we designed and implemented the LP32 code model such that the sizes of pointer and long are 32 bits. Our experiments demonstrate that on average the LP32 code model speeds up the SPEC2000 C benchmarks by $13.4 \%$. For the register-based argument passing convention, our experiments show that the performance gain is less than $1 \%$ because of the aggressive function inlining optimization. Finally, we observe that using 16 GPRs and 16 XMM registers significantly outperforms the scenario when only 8 GPRs and 8 XMM registers are used. However, our results also show that using 12 GPRs and 12 XMM registers can achieve as competitive performance as employing 16 GPRs and 16 XMM registers.
\end{abstract}

\section{Introduction}

In year 2003, AMD first introduced the AMD64 Opteron processor family that extends the existing 32-bit x86 architecture to 64-bit. In the following year, Intel announced that the Intel Extended Memory 64 Technology (EM64T) would be added to a series of IA-32 processors, which includes the Pentium 4 and Pentium M processors. Both AMD's AMD64 architecture and Intel's EM64T architecture allow applications to access up to one terabyte of memory address space. In addition, 64-bit Windows and Linux operating systems running on 64-bit x86 processors are supported by Microsoft, Red Hat and SuSE. Intel's 64-bit extension technology software 
developer's guide [1] details the EM64T architecture and the programming model. With the advent of 64-bit x86-compatible processors, it is imperative to understand its performance potential and its capability. Although the experiments of this study are done on the EM64T machine, we believe many of the results and insights can be applied to the AMD64 machine. (Note that EM64T and AMD64 are nearly binary compatible with each other, except for some of the instructions like AMD's 3DNOW and Intel's SSE3.)

The 64-bit x86 architecture furnishes many advantages over the 32-bit x86 architecture. The main advantages include: (1) 64-bit pointers over 32-bit pointers. 64-bit pointer allows applications to get access to one terabyte address space directly; (2) 64bit-wide general purpose registers (GPRs) over 32-bit-wide GPRs. The 64-bit-wide GPR allows 64-bit integer operations to be executed natively; and (3) 16 GPRs and 16 XMM registers over 8 GPRs and 8 XMM registers. With more registers, more values can be put into registers instead of in memory, and thus improve the performance. However, on the other side, we noticed that the 64-bit x86 architecture does not favor all the applications. For instance, within the 64-bit environment, some system vendors run 32-bit applications to report the peak performance, and a prior experiment [15] discusses several SPEC benchmarks whose 32-bit code can run substantially faster than their 64-bit counterparts can.

In this paper, we evaluate how these new features provided by the 64-bit x86 architecture can affect applications' performance from compiler optimizations' perspective. The contributions of this paper are threefold. First, we implemented the LP32 code model that supports 32-bit-wide long and pointer data types, and measured the performance impact of the LP32 code model. Secondly, we compared the performance difference using two different argument passing conventions. Lastly, we studied the sensitivities of applications' performances with respect to the number of allocatable registers.

The rest of the paper is organized as follows. In Section 2, we describe the infrastructure of the experimental framework. In Section 3, we evaluate the performance impact of the LP32 code model. In Section 4, we compare the register-based argument passing convention with the stack-based argument passing convention. In Section 5, we evaluate how applications' performances are improved with more allocatable registers. In Section 6, we briefly describe how the other 64-bit x86 features can improve application's performance. Finally, we conclude our study in Section 7.

\section{Experimental Framework}

In this section, we first give an overview of the 64-bit x86 architecture, and then illustrate how we set up the experimental infrastructure, which include how we construct a research compiler and how we characterize the performance impact of the 64-bit x86 architecture's new features.

\subsection{Overview of the 64-bit x86 Architecture}

The 64-bit $\mathrm{x} 86$ architecture is an extension of the 32-bit $\mathrm{x} 86$ architecture: it increases the virtual linear address space from 32 bits to 64 bits, and supports physical address space to 40 bits. The 64-bit x86 architecture supports three operating modes: (1) 
legacy mode, which enables 32-bit operating system to run 32-bit software, (2) compatibility mode, which enables 64-bit operating system to run 32-bit legacy software without any modification, and (3) 64-bit mode, which enables the 64-bit operating system to run 64-bit software that can get access to the 64-bit flat linear address space. Only software running on 64-bit mode can get access to the extended registers, and directly address up to one terabyte of memory space.

Evolved from the IA-32 architecture, the 64-bit x86 architecture doubled the number of GPRs and XMM registers from 8 to 16, respectively. In addition, the width of GPRs is extended from 32 bits to 64 bits. Equipped with more registers, the argument passing convention is changed considerably: instead of passing arguments through stack, now six GPRs and six XMM registers are dedicated to pass arguments [8].

In spite of rendering attractive new features, the 64-bit architecture comes at the cost of enlarging memory footprint. In both Linux and Windows operating systems, the size of pointer data type is widened from 32 bits to 64 bits, and in Linux operating system, the size of long data type is widened from 32 bits to 64 bits. Enlarged data types could degrade the performance of pointer intensive applications, because now they have bigger memory footprint that causes more cache misses. As another side effect of 64-bit address, occasionally sign extensions are required to perform address computations through 32-bit signed operands. In addition, the code size could increase due to a set of prefixes are introduced when extended registers or 64-bit register operands are referred. We will present additional details in the following related sections. In this paper, we conducted all the experiments on a Dell Precision Workstation 370 MiniTower containing a $3.8 \mathrm{GHz}$ Intel Pentium 4 processor. The system's detail configuration is listed in Table 1 .

Table 1. Hardware system configuration

\begin{tabular}{|l|l|}
\hline Processor & one 3.8 GHz Intel Pentium 4 Prescott \\
\hline L1 Trace cache & $\begin{array}{l}\text { 12K micro-ops, 8-way set associative } \\
\text { 6 micro-ops per line }\end{array}$ \\
\hline L1 Data cache & $\begin{array}{l}\text { 16KB, 4-way set associative } \\
\text { 64B line size, write-through }\end{array}$ \\
\hline L2 Unified cache & $\begin{array}{l}\text { 8-way, Sectored, 1MB unified L2 Cache } \\
\text { 64 byte line size }\end{array}$ \\
\hline Data TLB & fully associative, 4K/2M/4M pages, 64 entries \\
\hline Instruction TLB & 4-way associative, 4K pages, 64 entries \\
\hline System chipset & Intel $\mathbb{B}$ E7520 Chipset \\
\hline System bus speed & 800 MHz system bus \\
\hline Main memory & 3GB, 400MHz, DDR2 NECC SDRAM memory \\
\hline
\end{tabular}

\subsection{Methodology on Performance Characterization}

We used a research compiler to generate different executables to reflect the interested 64-bit x86 architectural features. Once the executables were generated, we applied a set of performance profiling tools to study the performance characteristics. The following sections described the tools and the operating system that we used in this study. 


\subsubsection{Overview of the Intel Compiler}

Our research compiler is based on Intel's 9.0 version Fortran and C++ production compiler that supports many Intel architectures. A high-level overview of the Intel compiler infrastructure is given in Figure 1. In this study, we used options " $-O 3-x P-$ ipo -prof_use" to compile all the benchmarks. Here option $-x P$ specifies that the compilation is targeting for a Prescott family processor, option -ipo enables the use of inter-procedural optimization, and option -prof_use enables the use of profiling information during compilation. Since we started this study, a few new optimizations and enhancements have been implemented to the product Intel compiler. For this reason, our research compiler may not demonstrate the best performance, and thus we only present relative performance numbers in the experimental result sections.

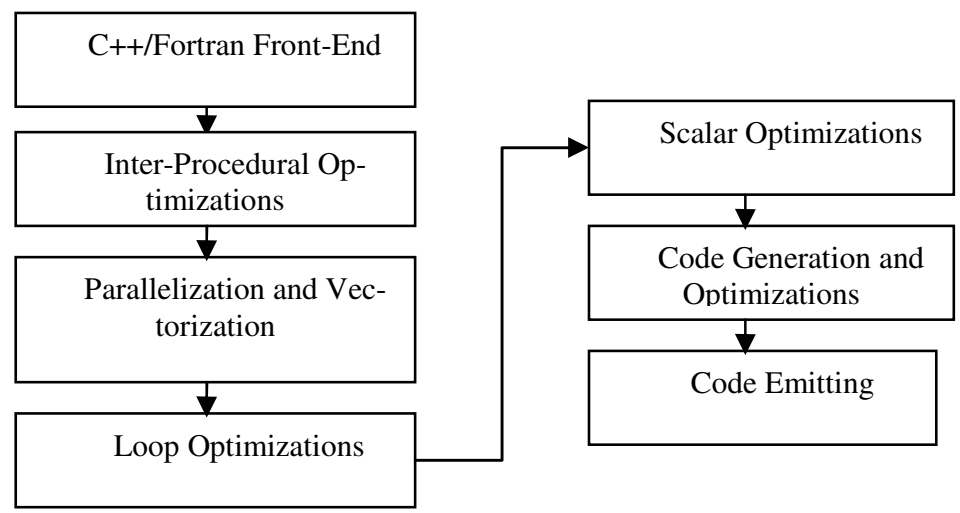

Fig. 1. A high-level overview of the Intel compiler architecture

Throughout this study, we conducted our experiments using the SPEC CPU2000 benchmark suite, which consists of twelve integer benchmarks (CINT2000) and fourteen floating-point benchmarks (CFP2000). In this paper, we refer all the SPEC2000 benchmarks that are written in C as SPEC $2000 \mathrm{C}$ benchmarks.

\subsubsection{Profiling Tools}

Intel's Pentium 4 processor provides a rich set of performance-monitoring counters that can be used for performance tuning [5][17]. In this study, we used emon, a lowlevel and low overhead performance tool, to collect the L2 cache references from the performance-monitoring counters by monitoring event BSQ_cache_reference. This event counts the number of second level cache loads and read-for-ownership (RFO) as seen by the bus unit. RFO happens when memory writes that miss must read the rest of the cache line to merge with new data.

Besides emon, we used pin [4] to collect the dynamic instruction distribution information. Pin is a dynamic instrumentation system developed at Intel Corporation. It provides a rich set of APIs so that users can write tools to analyze an application at the instruction level. Here we used a modified version of insmix, one of the pintools provided by pin, to the collect dynamic instruction information. 


\subsubsection{Operating System}

We conducted all our experiments on the Enterprise Linux operating system with kernel 2.6.9-5. We chose Linux operating system, because it provides us the flexibility to modify the kernel to support the LP32 code model.

To support the LP32 code model in this study, we modified the operating system kernel by specifying the value of macro TASK_SIZE to Ox100000000UL within file asm-x86_64/processor.h. In this manner, user applications' spaces are allocated within the lower 32-bit address space. (Note that modifying the value of TASK_SIZE to support the LP32 code model might not be the best approach. We adopted this approach to serve our evaluation purpose only.)

\section{Performance Characterization of the LP32 Code Model}

Under the 64-bit processor environment, the sizes of long and pointer data types by default are 64 bits on Linux operating system. In this study, we implemented the LP32 code model so that the sizes of long and pointer are 32 bits. To illustrate the differences among different modes, Figure 2 depicts how the address of variable $j$ is stored into the $i$-th element of array $a, a[i]=\& j$, in 64-bit mode, compatibility mode, and the LP32 code model, respectively.

\begin{tabular}{|c|c|}
\hline 48630500000000 & movslq i(\%rip), \%rax \\
\hline $48 \mathrm{c} 704 \mathrm{c} 5000000$ & $\begin{array}{l}\operatorname{movq} \$ \mathrm{j}, \mathrm{a}(, \% \operatorname{rax}, 8) \\
\text { (a) }\end{array}$ \\
\hline a1 00000000 & movl i, \%eax \\
\hline c7 048500000000 & $\begin{array}{l}\operatorname{movl} \$ \mathrm{j}, \mathrm{a}(, \% \mathrm{eax}, 4) \\
\text { (b) }\end{array}$ \\
\hline $8 b 0500000000$ & movl i(\%rip), \%eax \\
\hline 67 c7 0485000000 & $\operatorname{movl} \$ \mathrm{j}, \mathrm{a}(, \%$ eax, 4$)$ \\
\hline
\end{tabular}

(c)

Fig. 2. Different code sequences in different modes used to perform assignment " $a[i]=\& j$ ". (a) Instruction sequence generated in 64-bit mode. (b) Instruction sequence generated in compatibility mode. (c) Instruction sequence generated in the LP32 code model.

As depicted in Figure 2(a) and Figure 2(b), the differences between compatibility mode and 64-bit mode are threefold: (1) application memory footprint is increased for larger pointer; (2) sign extension operation is required to compute the 64-bit address; and (3) code size is increased because of prefixes. In Figure 2(c), address-size prefix $0 x 67$ is required to specify that the effective address size is 32 bits. (In 64-bit mode, the default effective address size is 64 bits; in compatibility mode, the default effective address size is 32 bits.) In this section, we study how the aforementioned modes could affect the SPEC2000 C benchmarks' performance.

\subsection{Implementation Challenges}

We encountered numerous challenges during the implementation of the LP32 code model. The first challenge was how to guarantee that the user stack are allocated in 
the lower 32-bit address space. A quick work around is described in Section 2.2.3 by modifying the operating system kernel. Once that was resolved, we need to solve the compatibility issue: external functions do not understand the LP32 code model.

One solution is to provide a group of LP32 compatible libraries. In this study, we implemented a set of wrappers for those external functions that will read/write the user-defined pointer- or long-type data directly or indirectly. Across all the SPEC2000 C benchmarks, we experienced few such kind of external functions; typical instances are time(), fstat(), and times(). Note that the performances of these functions do not affect our characterization results.

\subsection{Experimental Results}

Figure 3 shows the normalized execution time (with respect to base compilation in 64-bit mode) of the ILP32 code model, compatibility mode, and the LP32 code model. The ILP32 code model is the result of the auto ilp32 optimization, which will be elaborated in Section 3.3. On average, the LP32 speedups the performance across all the SPEC C benchmarks by $13.4 \%$. Without otherwise stated, we use geometric mean to calculate the average values.

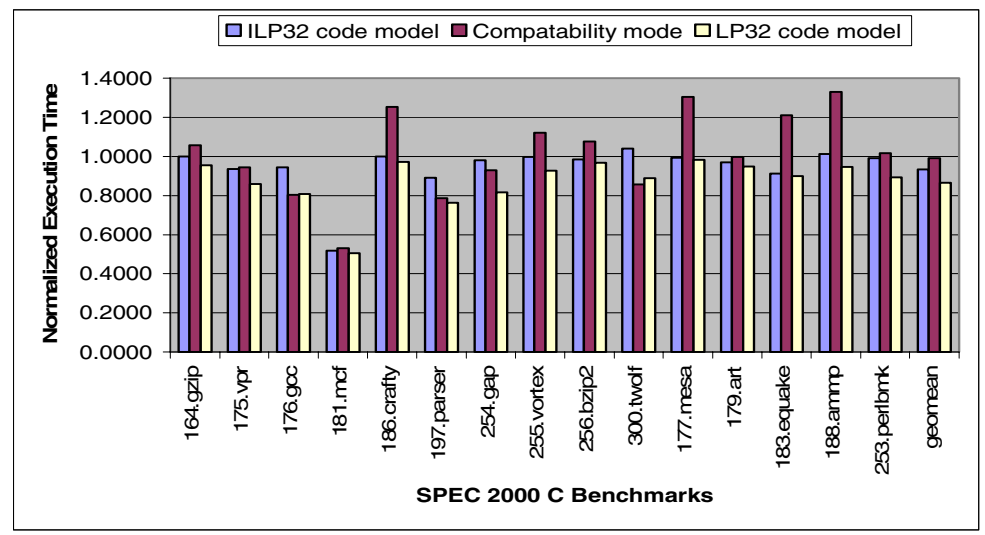

Fig. 3. The normalized execution time in the ILP32 code model, compatibility mode, and the LP32 code model for the SPEC2000 C benchmarks

Figure 4 gives the normalized L2 cache miss ratio for the SPEC2000 C benchmarks in the LP32 code model. Since the out-of-order execution engine of the 64-bit x 86 processor can effectively hide many of stalls incurred by cache misses, the purpose of this figure is not to correlate with Figure 3 directly, but to provide us some insights on how the LP32 code model can reduce the cache miss ratio. Notice that the L2 cache miss ratio of benchmark 183.equake is increased by more than $20 \%$. This is because in the LP32 code model, the absolute numbers of L2 cache accesses and L2 cache misses are reduced, yet the number of $\mathrm{L} 2$ cache accesses is reduced more.

Across the SPEC2000 C benchmarks, 181.mcf gains the most speedup, by almost $50 \%$. This is understandable because 181 .mcf is a memory-bound benchmark, and 


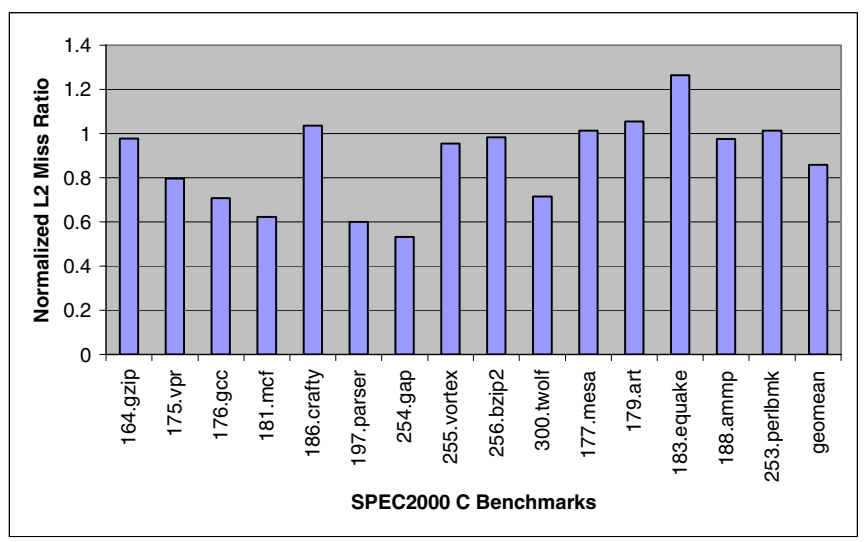

Fig. 4. The normalized L2 cache miss ratio for the SPEC2000 C benchmarks in the LP32 code model

many of the cache optimization techniques can improve its performance greatly. However, not all the performance loss in 64-bit mode is due to enlarged memory footprint. Benchmark 254.gap exemplifies our argument. Compiled in the LP32 code model, the 254.gap benchmark demonstrates a $20 \%$ speedup. We observed that the performance of this benchmark is dominated by a garbage collection function named CollectGarb(). This function will be invoked when the internal memory pool is exhausted. In compatibility mode, CollecGarb() is invoked 74 times, as opposed to 132 times in 64-bit mode. This is because some objects allocated within 254.gap require more memory space in 64-bit mode than in compatibility mode, and thus the internal memory pool is used up more quickly. In fact, the execution time of 254.gap can be reduced by almost $10 \%$ if we double the memory space allocated to its internal memory pool.

\subsection{Related Work}

For 32-bit applications, the LP32 code model is an effective approach to reduce the memory footprint, and leverage the new hardware features. However, in practice, it requires tremendous engineering effort to maintain a separate set of libraries and part of the kernel layer support.

To reduce the enlarged memory footprint in 64-bit mode, gcc compiler and pathcc compiler can generate 32-bit executables if users specify $-m 32$ compilation option, yet at the cost that these executables can only be executed in compatibility mode. To overcome this limitation, Intel compiler provides auto ilp32 optimization, which can represent the sizes of pointer and long data type values in 32 bits, and the applications can use all the new features of the 64-bit x86 architecture. However, the current optimization scope of the auto ilp32 optimization is limited: it only considers pointers within objects allocated by malloc() function, and its effectiveness relies on the accuracy of the points-to analysis. The auto ilp32 optimization can be regarded as a tradeoff solution between the $-m 32$ mode and the LP32 code model.

Recently, Lattner and Adve use pointer compression technique to reduce the memory space consumed by 64-bit pointers for linked list data structure [9]. The idea is to 
allocate dynamically created linked list objects in the contiguous memory space, and replace the original 64-bit pointers with 32-bit integer values that can be used as offsets. The original pointer value can be calculated through this offset and the starting address of the memory pool. This approach requires whole program analysis and introduces run-time overhead for pointer compression and decompression. Similar approach is adopted by Intel's open runtime platform [10] that compresses 64-bit-wide pointers in a similar way for applications written in Java language.

\section{Register Argument Passing Convention}

Argument passing convention specifies how arguments are passed from caller function to callee function. In compatibility mode, arguments are passed through stack. In 64-bit mode, leveraged with doubled registers, six GPRs are dedicated to pass integer arguments, and six XMM registers are dedicated to pass floating-point arguments [8]. The major benefit of register argument passing convention is to reduce the stack accesses, and thus improve the performance, especially for call-intensive applications.

In this section, we compare the performance difference between the stack-based argument passing convention and the register-based argument passing convention in 64-bit mode.

\subsection{Implementation Challenges}

In this study, we implemented two argument passing schemes within the compiler: (1) arguments are passed through registers, which is the default convention in 64-bit mode; and (2) arguments are passed through stack, which is the default convention in compatibility mode. For the purpose of performance study, we implemented the later convention scheme for the compilation in 64-bit mode.

To emulate the stack-based argument passing convention in the 64-bit environment, it is compiler's responsibility to maintain the convention compatibility between caller and callee functions: if the callee function is an external function that is not processed by compiler (i.e., library functions), then the arguments should be passed through registers at the call site. Likewise, if the caller function is an external function, then the callee function should expect the arguments are passed through registers. Under other scenarios, arguments are passed through stack. To preserve the compatibility, we relied on the information provided by the inter-procedural optimization (IPO), which will process all the user-defined functions before argument passing convention code is generated.

Empirical study shows that only a handful of functions within the SPEC2000 need to use the default convention, and most of these functions are called by external function $q \operatorname{sort}()$, which is a library function that will call user-defined functions for data comparisons. Execution time profiling information shows that these functions do not have noticeable performance impact.

\subsection{Experimental Results}

Figure 5 shows the normalized execution time (with respect to the base compilation using register argument passing convention) when the stack-based argument passing 
convention is applied for the SPEC2000. On average, the CINT2000 is slowed down by $0.86 \%$, and almost no noticeable slowdown for the CFP2000. On the contrary, we can see that some of the benchmarks actually have better performance with stackbased convention. For example, benchmark 300.twolf has an almost $4 \%$ speedup. However, 300.twolf is a volatile benchmark, and it is difficult to reproduce the result consistently.

Curious by the marginal performance difference, we suspected that the advantages of using registers to pass arguments were mainly obscured by the aggressive function inlining optimization, which potentially eliminates many of the function calls. To confirm our hypothesis, we turned off function inlining and partial function inlining optimizations by specifying compilation option “-ip-noinlining -ip-no-pinlining”. The normalized execution time without functional inlining or partial inlining for the SPEC2000 is given in Figure 6. We can see a clear performance difference: the CINT2000 has a 7.4\% performance slowdown and the CFP2000 has a $1.2 \%$

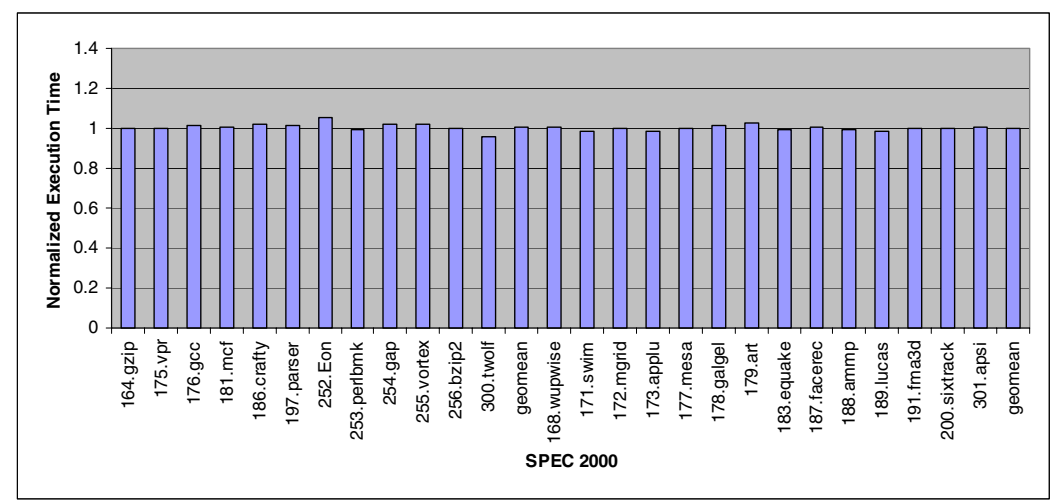

Fig. 5. The normalized execution time when the stack-based argument passing convention for the SPEC 2000

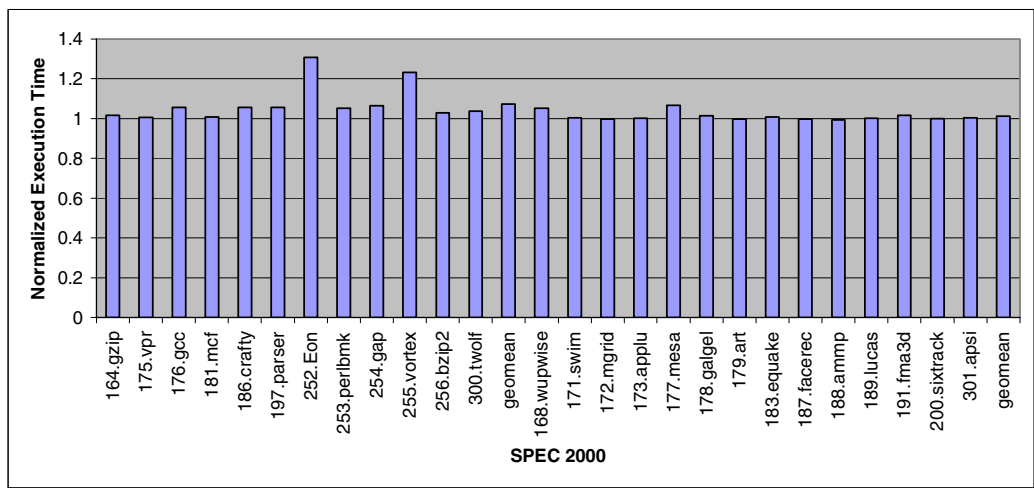

Fig. 6. The normalized execution time when the stack-based argument passing convention for the SPEC2000 with function inlining and partial inlining being turned off 
performance slowdown. Among all the benchmarks, 252.eon and 255.vortex slow down the most, by $31 \%$ and by $23 \%$, respectively. For 252 .Eon benchmark, the number of dynamic function calls is increased by a factor of 86 if the function inlining optimization is turned off.

\subsection{Related Work}

To our best knowledge, there is no direct performance comparison between these two different argument passing conventions. Hubička [3] hypothesized that the register argument passing convention could improve the performance. However, our experiment shows that the performance impact is very limited, given that most of function calls have been eliminated by a sophisticated function inlining optimization.

\section{Performance Improvement with More Registers}

Register allocation has long been considered as one of the most important optimization techniques conducted by modern compilers. Alleviated with more registers, compiler can keep more values in registers without going through the memory system, and thus speed up application's performance. The 64-bit x86 architecture provides 16 GPRs (including the stack pointer register) and 16 XMM registers, which is twice as many registers that IA-32 architecture provides.

However, introducing more registers also brings in new challenges. First, the code size could be enlarged. Whenever an extended register or a 64-bit operand is refered, the REX prefix is required as the first opcode byte of the instruction [16]. These extra REX prefixes increase instruction size, and thus code size. Second, more caller-saved registers could affect performance. In the IA-32 processor, there are total 11 callersaved registers (including 3 GPRs and 8 XMM registers). The number is more than doubled in the 64-bit $x 86$ architecture, which has total 25 caller-saved registers (including 9 GPRs and 16 XMM registers). Saving more caller- and callee-saved registers could degrade performance, and increase the code size.

In this section, we study how the number of memory accesses is reduced with more registers, and how applications' performances are affected by varying the number of allocable registers in 64-bit mode. Instead of experimenting with all the different combinations, here we focus on two set of register configurations: (1) $R E G \_8$, where 8 GPRs and 8 XMM registers can be allocated by register allocator, and (2) $R E G \_12$, where 12 GPRs and 12 XMM registers can be allocated by register allocator.

\subsection{Implementation Challenges}

We conducted this study by adjusting the number of available registers can be allocated by the register allocator within the compiler. The register allocation algorithm implemented by the research compiler is an extension of the Chaitin-Briggs style graph coloring algorithm [6][7].

It is a straightforward process to setup the number of available registers for the register allocator. However, in 64-bit mode, register $R 8$ and register $R 9$ will be used to 
pass the fifth and the sixth integer arguments, respectively. To comply with the argument passing convention, when the available GPRs do not include R8 and R9, we have to deploy R8 and R9 specifically for integer argument passing purpose only, if they are required.

\subsection{Experimental Results}

Figure 7 gives the normalized execution time (with respect to the base compilation using 16 GPRs and 16 XMM registers) with the REG_8 and REG_12 register configurations for the SPEC2000 benchmarks. On average, with the REG_8 configuration, the CINT2000 exhibits a 4.4\% slowdown, and the CFP2000 exhibits a 5.6\% slowdown; with the REG_12 configuration, the CINT2000 is slowed down by $0.9 \%$, and the CFP2000 is slowed down by $0.3 \%$. Clearly, these results show that REG_12 already works well for most of the SPEC2000 benchmarks.

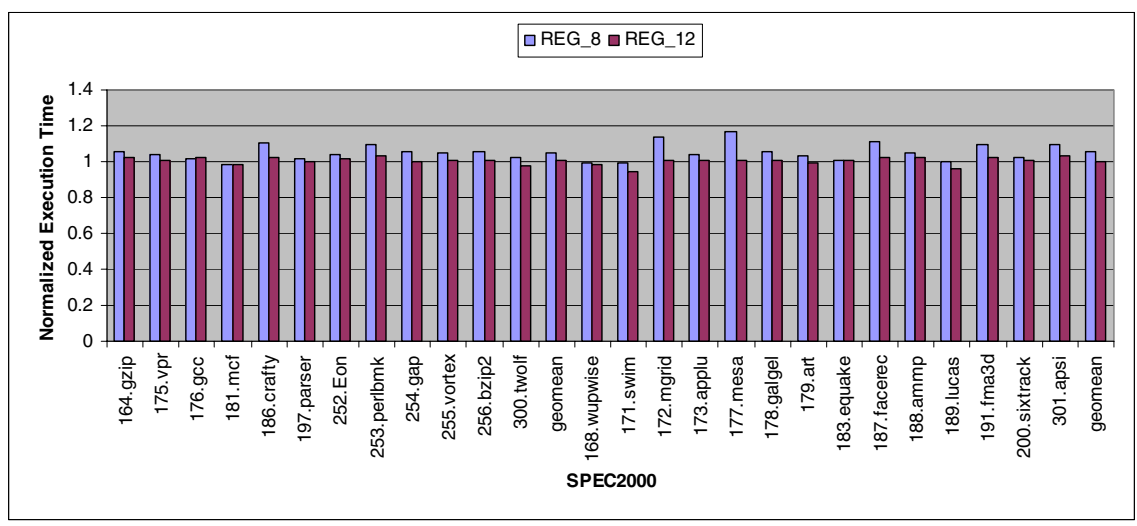

Fig. 7. The normalized execution time for the SPEC 2000 benchmarks with the REG_8 and REG_12 configurations

Note that REG_8 slightly outperforms REG_12 on 176.gcc benchmark. In addition, for 181.mcf and 300.twolf benchmarks, the fewer the registers, the better the performances are. (We do not pursue the cause further, since the improvement with fewer registers is only within the $2 \%$ range.)

In addition to performance, the normalized dynamic number of memory accesses (including stack accesses) for the CINT2000 and CFP2000 is shown in Fig. 8. On average, with the REG_8 configuration, the memory references are increased by $42 \%$ for the CINT2000 and by 78\% for the CFP2000; with the REG_12 configuration, the memory references are increased by $14 \%$ for CINT2000 and by $29 \%$ for the CFP2000.

Overall, for the SPEC2000 benchmark suite, we can see the trend that when the number of available registers is increased from 8 to 12 , the memory accesses are reduced dramatically, and moderate performance improvement is achieved. However, with more than 12 registers, although the memory accesses are further reduced, the performance is barely improved. 


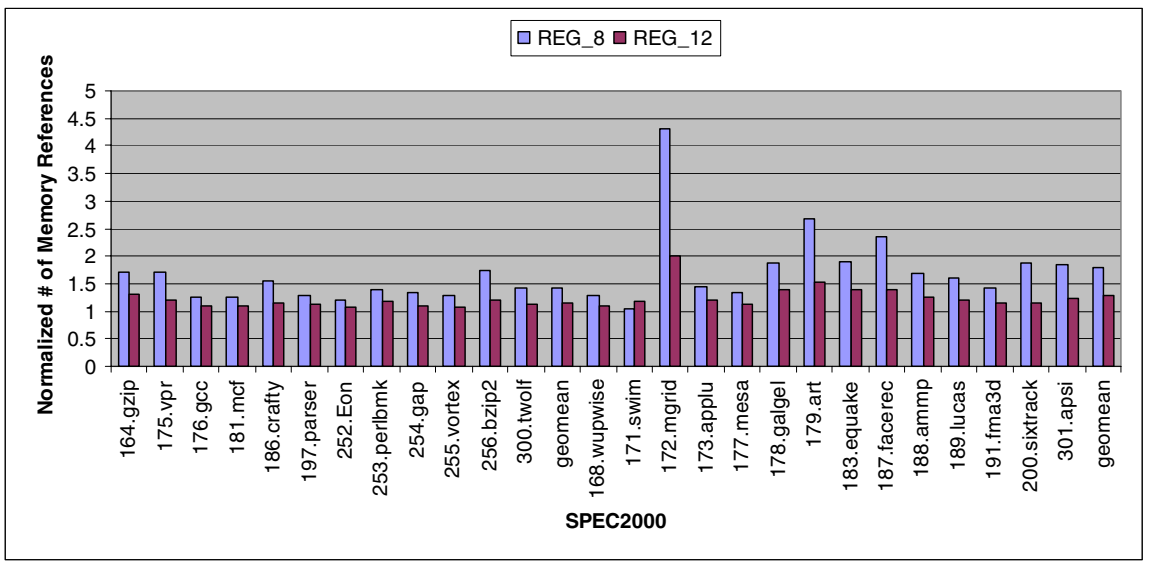

Fig. 8. The normalized number of memory accesses for the SPEC 2000 benchmarks with the REG_8 and REG_12 configurations

Once the number of allocatable registers reaches a certain threshold, results show that the performance cannot be improved further even given more registers. We suspected that this phenomenon is due to the powerful out-of-order execution engine of the $x 86$ core. In addition, comprehensive compiler optimization techniques can reduce the register pressure by folding some of the memory load instructions into other instructions as operands.

\subsection{Related Work}

An abundant research has been conducted on register allocation. Optimal, or nearoptimal, register allocation algorithms [11][13][14] are also proposed for IA-32 architecture. Most of the prior works only demonstrate how the amount of spill code can be reduced and how efficient the solvers are (while compared with an exponential approach). However, not much work has been done on experimenting with the recent SPEC benchmark suite to demonstrate how the additional registers could improve the application's performance.

Luna et al. [2] experimented with different register allocators on AMD64 platform. Their studies show that with more registers, a fast linear scan based register allocation algorithm [18] could produce competitive performance code with graph coloring based algorithm. It is interesting for us to evaluate the performance of a linear scan approach with different number of registers on the SPEC2000. In addition, Luna et al. show that the code size could be increased by $17 \%$ due to REX prefixes.

Experimented on an out-of-order MIPS processor, Govindarajan et al. [12] propose an instruction scheduling algorithm to reduce the spill code. Their experiment on SPEC95 floating-point benchmarks show that their technique could reduce the average number of spill code by $10.4 \%$ and $3.7 \%$, respectively, and on average the performance is improved by $3.2 \%$. Note that MIPS architecture has 32 GPRs, as compared with 16 GPRs supported by the 64-bit x86 architecture. 


\section{Other 64-bit x86 Architecture Features}

One important feature of the 64-bit $x 86$ architecture is that the width of GPR is increased from 32 bits to 64 bits. In this study, we do not explicitly investigate the effectiveness of wider GPRs. From our experience, 197.crafty is the only benchmark that gains most of the benefit, a $20 \%$ speedup, from 64-bit-wide GPRs. This is because 197.crafty performs intensive arithmetic operations on long long type data. Without 64-bit GPRs, sequences of 32-bit operations will be expanded to perform simple 64-bit operations. The example depicted in Fig. 9 illustrates this point.

$$
\begin{array}{ll}
\text { movl } & \mathrm{i}, \% \text { edx } \\
\text { movl } & 4+\mathrm{i}, \% \text { ecx } \\
\text { addl } & \mathrm{j}, \% \text { edx } \\
\text { adcl } & 4+\mathrm{j}, \% \text { ecx } \\
\text { movl } & \% \mathrm{edx}, \mathrm{k} \\
\text { movl } & \% \text { ecx, } 4+\mathrm{k}
\end{array}
$$

(a)

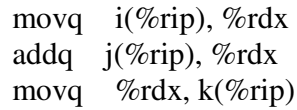

(b)

Fig. 9. Example illustrating the code generations for assignment " $k=i+j$ ", where $k, i$ and $j$ are long long data type variables. (a) Instructions generated in compatibility mode. (b) Instructions generated in 64-bit mode.

In this example, it takes three 64-bit instructions to perform a 64-bit add operation " $k=i+j$ " in 64-bit mode. However, it requires six 32-bit instructions to do the same task in compatibility mode: two load instructions are used to load the 64-bit value of variable $i$ to two 32-bit registers, two add instructions are used to perform a 64-bit add operation using 32-bit operands, and two store instructions to store the 64-bit value to variable $k$.

In addition, the 64-bit $x 86$ architecture introduces a new instruction-pointer relative-addressing mode, which can reduce the number of load-time relocations for position-independent code while accessing global data. A prior work [3] demonstrates that relative-addressing mode can reduce the performance loss significantly for positionindependent code from $20 \%$ down to $6 \%$.

\section{Conclusions and Future Works}

With the emerging of the 64-bit x86 architecture, it is imperative to understand its capability and its limitation. In this study, we evaluate several interesting new features of the 64-bit x86 architecture using a production level research compiler and conducting our experiments on the SPEC2000 benchmark suite.

The most noticeable change from 32-bit x86 to 64-bit x86 is that the size of pointer is increased from 32 bits to 64 bits. Experimenting on the SPEC2000 C benchmarks, we observe that the widened pointer and long data types could slow down an application's performance by as much as 50\%. Prior studies on IA-32 processors [19][20] show that the number of data cache misses plays a critical role on modern database management systems (DBMS). The situation is even more severe in the 64-bit x86 processors that inherit larger memory footprint. It is interesting to see how the LP32 
code model can help here. Besides studying 64-bit pointer, we also evaluate how the register-based argument passing convention could improve performance. Experiments show that the performance gain is imperceptible if an aggressive function inlining optimization is performed. Finally, we evaluate how the double sized register file could improve the performance. Experimental results show that using 12 GPRs and 12 $\mathrm{XMM}$ registers can achieve almost the same performance as using 16 GPRs and 16 $\mathrm{XMM}$ registers can.

The above observations point our future research to the following three areas: (1) investigate how to reduce memory footprint in the 64-bit x86 processors on DBMS; (2) investigate and improve the performance of linear scan register allocation [18]; and (3) study the interaction between register allocation and instruction scheduling in the 64-bit x86 processors.

\section{Acknowledgements}

We thank Suresh Siddha for the advice on modifying the Linux kernel to support the LP32 code model, Somnath Ghosh for explaining the auto ilp32 optimization implemented in the Intel production compiler, Robert Cohn for the help in resolving a couple of PIN issues due to the modified Linux kernel, Thomas Johnson for the suggestions on using the emon tool, and Jin Lee for providing us the experimental machine. We also thank many anonymous reviewers for their helpful comments on earlier drafts of this paper.

\section{References}

[1] Intel Corporation, Santa Clara. "64-bit Extension Technology Software Developer's Guide Volume 1\&2" Order Number 300834, 300835

[2] Daniel Luna, Mikael Pettersson, and Konstantinos Sagonas. "Efficiently compiling a functional language on AMD64: the HiPE experience," PPDP '05: Proceedings of the 7th ACM SIGPLAN international conference on Principles and practice of declarative programming, 2005, pages 176--186

[3] Jan Hubička, "Porting GCC to the AMD64 architecture," In Proceedings of the GCC Developers Summit, pages 79--105, May 2003

[4] Chi-Keung Luk, Robert Cohn and et al. "Pin: building customized program analysis tools with dynamic instrumentation," In proceedings of the ACM SIGPLAN 2005 Conference on Programming language design and implementation, Pages 190-200, 2005

[5] Brinkley Sprunt. "Pentium 4 Performance-Monitoring Features," IEEE Micro, Vol 22, Num 4, 2002, Pages 72-82

[6] G. J. Chaitin. "Register allocation \& spilling via graph coloring," SIGPLAN '82: Proceedings of the 1982 SIGPLAN symposium on Compiler construction, 1982, Pages 98-101

[7] P. Briggs, K. D. Cooper, K. Kennedy and L. Torczon. "Coloring heuristics for register allocation," PLDI '89: Proceedings of the ACM SIGPLAN 1989 Conference on Programming language design and implementation, pages 275-284

[8] Ed. J. Hubička, A. Jaeger and M. Mitchell. "System V Application Binary Interface: AMD64 Architecture Processor Supplement.” Available from www.x86-64.org.

[9] Chris Lattner and Vikram S. Adve. "Transparent Pointer Compression for Linked Data Structures,” In Proceedings of Memory System Performance Workshop, 2005 
[10] Ali-Reza Adl-Tabatabai et al., "Improving 64-Bit Java IPF Performance by Compressing Heap References," In Proceedings of CGO, pages 100_111, March 2004

[11] David Koes and Seth Copen Goldstein. "A Progressive Register Allocator for Irregular Architectures," In Proceedings of CGO, pages 269--280, 2005

[12] R. Govindarajan, Hongbo Yang, Jose Nelson Amaral, Chihong Zhang and Guang R. Gao. "Minimum Register Instruction Sequencing to Reduce Register Spills in Out-of-Order Issue Superscalar Architectures," IEEE Transaction on Computers, 52(1), January 2003.

[13] Timothy Kong and Kent D. Wilken. "Precise register allocation for irregular architectures," In Proceedings of the 31st annual ACM/IEEE international symposium on Microarchitecture, pages 297-307, 1998

[14] Andrew W. Appel and Lal George. "Optimal spilling for CISC machines with few registers," In proceedings of the ACM SIGPLAN 2001 conference on Programming language design and implementation, pages 243-253, 2001

[15] Kirill Kochetkov, "SPEC CPU2000. Part 19. EM64T in Intel Pentium 4," June 2005. Available from http://www.digit-life.com/articles2/cpu/insidespeccpu2000-part-j.html.

[16] Intel Corporation, Santa Clara. "IA-32 Intel® Architecture Software Developer's Manual, Volume 1: Basic Architecture," 2005. Order Number 253665.

[17] Intel Corporation, Santa Clara. "IA-32 Intel® Architecture Software Developers Manual, Volume 3: System Programming Guide," 2005. Order Number 253668.

[18] Massimiliano Poletto and Vivek Sarkar, "Linear scan register allocation," ACM Transactions on Programming Languages and Systems, 21(5), pages 895—913, 1999

[19] Anastassia Ailamaki, David J. DeWitt, Mark D. Hill, David A. Wood, "DBMSs on a Modern Processor: Where Does Time Go?" Proceedings of 25th International Conference on Very Large Data Bases, pages 266-277, September 1999, Edinburgh, Scotland, UK

[20] Kimberly Keeton, David A. Patterson and et al., "Performance characterization of a Quad Pentium Pro SMP using OLTP workloads," In Proceedings of the 25th annual international symposium on Computer architecture, pages 15-26, 1998 